\title{
Changes in muscle force following therapeutic electrical stimulation in patients with complete paraplegia
}

\author{
H Kagaya, Y Shimada, K Sato and M Sato \\ Department of Orthopedic Surgery, Akita University School of Medicine, 1-1-1 Hondo, Akita 010, Japan
}

\begin{abstract}
Changes in muscle force following therapeutic electrical stimulation (TES) in 5 patients with complete paraplegia were evaluated by computed tomography, Cybex II, a strain-gauge transducer, and manual muscle testing. The stimulation parameters included a frequency of $20 \mathrm{~Hz}$, a pulse width of $0.2 \mathrm{~ms}$, and an output voltage of $-15 \mathrm{~V}$. The cross-sectional areas of muscles, the muscle torques, and the muscle forces increased after TES, though the increased ratio differed in each muscle. The reasons why it differed are as follows: (1) The possibility of peripheral nerve injury; (2) The different patterns of nerve distribution in the muscles; (3) Implantation techniques; and (4) The possibility of breakage, movement, or changes in the impedance of electrodes. This study demonstrates that TES increases muscle force during electrical stimulation in paralyzed muscle, but that an initial TES-induced muscle force greater than a poor-minus level on manual muscle testing is necessary for practical use. TES treatment should be started as soon as possible after the onset of paralysis, in order to maintain muscle quality.
\end{abstract}

Keywords: therapeutic electrical stimulation (TES); functional electrical stimulation (FES); paraplegia; muscle force; spinal cord injury; spinal cord disease

\section{Introduction}

Recent development of functional electrical stimulation (FES) has allowed restoration of the motor function of paralyzed extremities. FES, using percutaneous electrodes, has been available in our department since 1990 . Our interest in this treatment is primarily the restoration of standing and walking in those with complete paraplegia. ${ }^{1}$

Therapeutic electrical stimulation (TES) is usually performed before FES as a muscle strengthening program, because atrophied muscles following paralysis cannot produce enough power for functional use. Electrical stimulation is a well-documented clinical method of augmenting muscle performance in both normal and paralyzed muscles. ${ }^{2-6}$ In complete paralysis, however, muscle force only during electrical stimulation is of value. Because we need strong muscle force for functional use, especially in the lower extremities to sustain body weight, it is very useful to know whether or not electrically stimulated muscles can gain enough force for functional use. The aim of this study is to evaluate the changes in muscle force following TES in those with complete paraplegia and to predict the outcome of muscle force obtained by TES at the beginning of this stimulation treatment.

\section{Materials and methods}

The subjects were 5 patients with complete paraplegia who ranged in age from 19 to 68 years at the beginning of TES. Two of patients had arterio-venous malformation at the level of L2, but the affected cord level was unclear. The other three patients had spinal cord injuries at the T12, T6, and T8 levels resulting from traffic accidents. The time since injury was from 3 months to 5 years (Table 1). Cases 1, 2, and 3 had thoraco-lumbar region injuries and had the potential of having peripheral nerve damage. These patients are included in our study because the muscles did contract by electrical stimulation; Marsolais and Kobetic ${ }^{7}$ have reported that even the trace muscle can be used for stimulation when the muscle contracts.

The percutaneous electrode was a helical coil wound

Table 1 Subjects involved in the TES program

\begin{tabular}{|c|c|c|c|c|c|c|}
\hline Case & $\begin{array}{c}\text { Age } \\
\text { (years) }\end{array}$ & Sex & Diagnosis & $\begin{array}{l}\text { Level } \\
\text { of } \\
\text { injury }\end{array}$ & $\begin{array}{l}\text { Duration } \\
\text { from } \\
\text { onset }\end{array}$ & $\begin{array}{l}\text { Number } \\
\text { of } \\
\text { electrodes }\end{array}$ \\
\hline 1 & 68 & M & AVM & L2? & 5 years & 14 \\
\hline 2 & 22 & M & SCI & $\mathrm{T} 12$ & 4 months & 19 \\
\hline 3 & 61 & $\mathbf{M}$ & AVM & L2? & 2 years & 22 \\
\hline 4 & 19 & $\mathrm{M}$ & SCI & T6 & 3 months & 30 \\
\hline 5 & 24 & M & SCI & T8 & 1 year & 34 \\
\hline
\end{tabular}


from a Teflon-coated 19 strand stainless steel cable ${ }^{8,9}$ (SES114, Nippon Seisen, Japan). The cable consisted of SUS 316L type hard drawn stainless steel wires; the diameter of a single stainless steel wire was $25 \mu \mathrm{m}$. The diameter of the cable was about $0.4 \mathrm{~mm}$. The tips of the electrodes were deinsulated for applying the stimulus current to each muscle. The electrodes were percutaneously implanted by a needle through the skin, and placed in the motor points of the nerves and muscles. Stimulated nerves and muscles are given in Table 2. All electrodes were subcutaneously gathered, slightly to the lateral part of the mid-thigh (Figure 1). The electrodes were soldered on to small connectors 2 weeks after the implantation. We used the commercially available portable FES stimulator (CE1230, NEC, Japan) which had 30 output channels for stimulation. It measures $14.5 \times 8.9 \times 3.1 \mathrm{~cm}$ and weighs $360 \mathrm{~g}$. The rectangular pulse trains used consisted of a pulse width of $0.2 \mathrm{~ms}$, a pulse interval of $50 \mathrm{~ms}$, and an output voltage of $-15 \mathrm{~V}$. On time was $5 \mathrm{~s}$ that included a $1 \mathrm{~s}$ rise time and a $1 \mathrm{~s}$ fall time,

Table 2 Inserted nerves and muscles in each side

\begin{tabular}{|c|c|c|c|c|c|c|}
\hline & Case & 1 & 2 & 3 & 4 & 5 \\
\hline \multirow[t]{4}{*}{ Nerves } & Femoral n. & 1 & 1 & 1 & 1 & 1 \\
\hline & Superior gluteal $\mathrm{n}$. & 1 & 1 & 1 & 1 & 1 \\
\hline & Common peroneal $\mathrm{n}$. & 1 & 1 & 1 & 1 & 1 \\
\hline & Tibial $\mathrm{n}$. & & 1 & 1 & 1 & 1 \\
\hline \multirow[t]{9}{*}{ Muscles } & Paraspinal $\mathrm{m}$. & & & & 2 & 2 \\
\hline & Iliopsoas m. & & & 1 & 1 & 2 \\
\hline & Gluteus maximus $\mathrm{m}$. & 1 & 1 & 1 & 3 & 3 \\
\hline & Rectus femoris $\mathrm{m}$. & 1 & & 1 & 1 & 1 \\
\hline & Vastus lateralis $\mathrm{m}$. & & $1^{*}$ & 1 & 1 & 1 \\
\hline & Vastus medialis $\mathrm{m}$. & & & 1 & 1 & 1 \\
\hline & Long head of biceps femoris $\mathrm{m}$. & 1 & 2 & 1 & 1 & 1 \\
\hline & Medial hamstring $\mathrm{m}$. & & 1 & & & \\
\hline & Adductor magnus $\mathrm{m}$. & & & & & 1 \\
\hline \multicolumn{2}{|c|}{ Reference electrode } & 1 & 1 & 1 & 1 & 1 \\
\hline \multicolumn{2}{|c|}{ Total } & 7 & 10 & 11 & 15 & 17 \\
\hline
\end{tabular}

*only right side

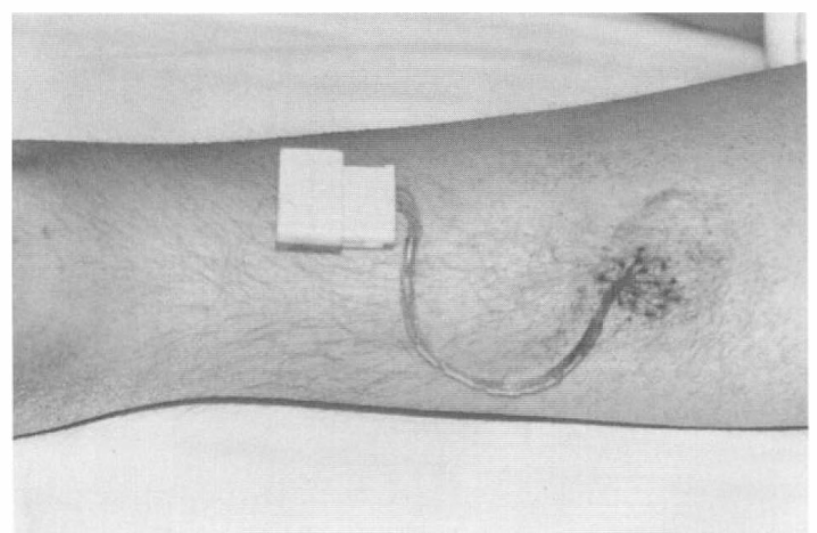

Figure 1 All electrodes were subcutaneously gathered, slightly to the lateral part of the mid-thigh and off time was $5 \mathrm{~s}$. The common peroneal nerve, the iliopsoas, the long head of the biceps femoris, and the medial hamstrings were stimulated simultaneously during the time when the other nerves and muscles were not stimulated. When the former were not stimulated, the latter were stimulated simultaneously. Stimulation of the muscles was for 10 min three times daily at the start, and was increased 10 min every 2 weeks. After 10 weeks, the stimulation was applied for $60 \mathrm{~min}$, three times daily. The stimulation period did not exceed $60 \mathrm{~min}$. TES continued for 6 months in either the hospital or in the home of the patient. The patients learned self-stimulation procedures.

Four tests were carried out to evaluate muscle force. These were as follows:

(1) Computed tomography (CT)

A CT (9200, YMS, Japan) scan was taken every 5 weeks at the upper edge of the femur head, at the parallel line $10 \mathrm{~cm}$ proximal to the knee joint, and at the parallel line $10 \mathrm{~cm}$ distal to the knee joint. In addition, the lower edge of the vertebral body at the 3 rd lumbar vertebra was scanned in patients 3,4 , and 5; electrodes were also inserted into iliopsoas and/or paraspinal muscles. We could examine patient number 4 five weeks prior to TES because we performed a posterior fusion (Luque SSI) at our hospital. The window level and window width were the same in each individual case, and the slice thickness was set at $10 \mathrm{~mm}$. The cross-sectional area of the gluteus maximus, the gluteus medius, the quadriceps femoris, the long head of the biceps femoris, the extensors in the lower leg, the iliopsoas, and the paraspinal muscles were determined with the help of a computer (PC9801RX, NEC, Japan), and the increased ratio was calculated as: the cross-sectional area after TES minus the cross-sectional area before TES, divided by the cross-sectional area before TES multiplied by $100(\%)$. The mean CT number of each muscle both before TES and 25 weeks after TES was calculated by computer (Dataview, YMS, Japan), and expressed in Houndsfield Units (HU).

(2) Muscle torque measured by Cybex II

The isometric muscle torque during electrical stimulation was measured periodically: with the knee flexed at 90 degrees for the knee extensors, the knee flexed at 60 degrees for the knee flexors, and the ankle plantarflexed at 30 degrees for the ankle dorsiflexors. Stimulating parameters consisted of a pulse width of $0.2 \mathrm{~ms}$, a pulse interval of $50 \mathrm{~ms}$, and an output voltage of $-15 \mathrm{~V}$.

(3) Muscle force measured with the help of a straingauge transducer.

The isometric muscle force during electrical stimulation was measured periodically, with the knee flexed at 90 degrees for the knee extensors and flexors. A straingauge transducer (9E01-L35-50K, NEC Sanei, Japan) was set $6 \mathrm{~cm}$ proximal to the medial malleolus of the 
ankle. The stimulating parameters were the same as above.

(4) Muscle force measured by manual muscle testing (MMT).

The muscle force during electrical stimulation was measured by MMT, and 13 grades were classified: normal, normal-minus, good-plus, good, good-minus, fair-plus, fair, fair-minus, poor-plus poor, poorminus, trace, and zero. ${ }^{10}$ MMT tests were carried out at the beginning of TES and 6 months later by the same physician. All stimulating parameters were the same as above.

\section{Results}

Figure 2 shows the changes in the increased ratio of the cross-sectional areas of muscles on the right side of case 4. The cross-sectional areas of all muscles increased after TES. In the iliopsoas muscle, the electrode broke 12 weeks after TES, at which time the cross-sectional area decreased.

All cross-sectional areas except the gluteus maximus generally increased during the time course. There was a significant difference between before TES and 25 weeks after TES using the paired $t$ test (in the gluteus medius, the quadriceps femoris, and the iliopsoas, $P$ was $<0.05$; in the paraspinal muscles, $P$ was $<0.01$; in the long head of the biceps femoris and the extensors in the lower leg, $P$ was $<0.001)$. The highest increased ratio was $107.7 \%$ and occurred on the right side of the long head of the biceps femoris in case 1 (Figure 3). On the other hand, the cross-sectional areas of the gluteus maximus muscles did not show a significant difference in cases 1,2 , or 3 , where only one electrode was inserted. The cross-sectional areas did show a significant increase $(P<0.05)$ using the paired $t$ test in cases 4 and 5 , where three electrodes were inserted in each muscle. The highest increased ratio in the gluteus maximus was $40.9 \%$ and occurred on the right side of case 5 (Figure 4).

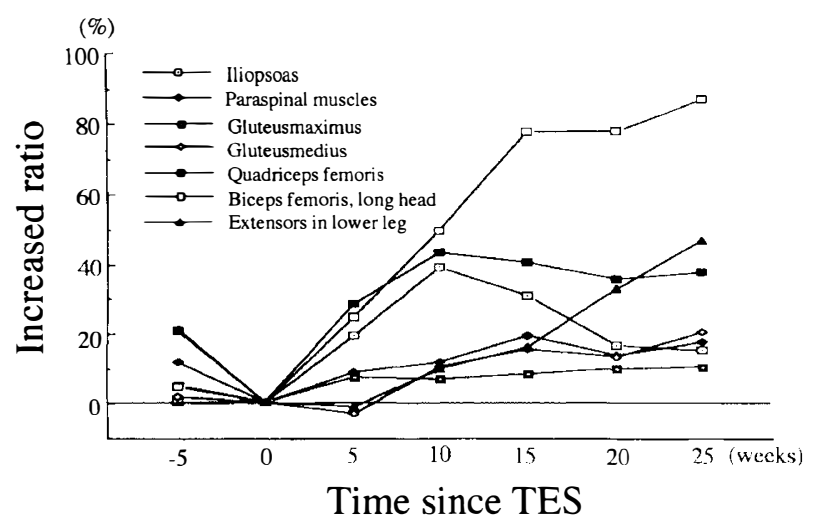

Figure 2 Changes in the increased ratio of the crosssectional areas of muscles on right side in case 4

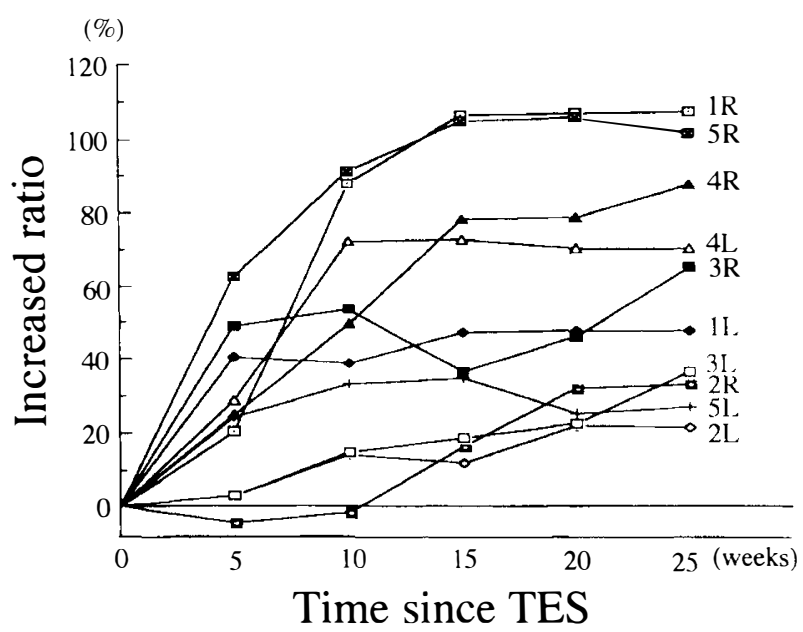

Figure 3 Changes in the increased ratio of the crosssectional areas of the long head of biceps femoris muscles. The cross-sectional areas increased significantly (paired $t$ test, $P<0.001)(1 \mathrm{~L}=$ casel, left. $1 \mathrm{R}=$ casel, right $)$

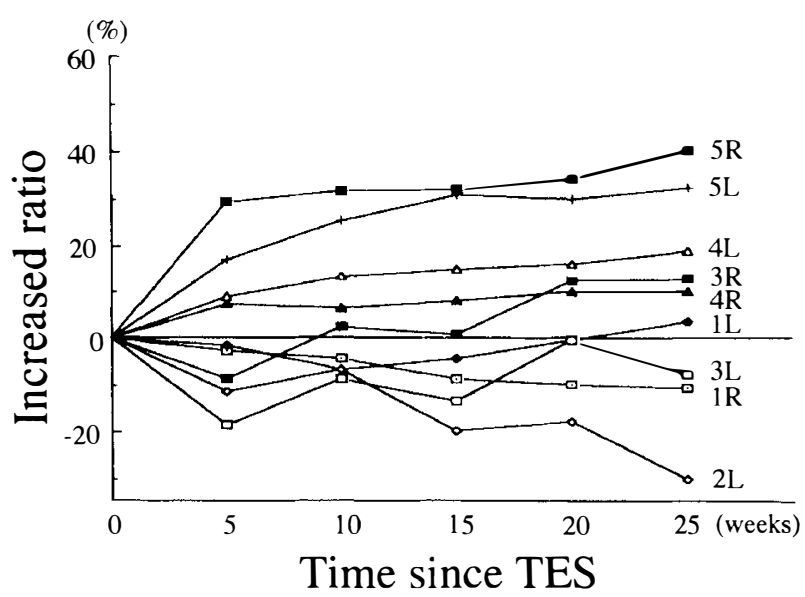

Figure 4 Changes in the increased ratio of the crosssectional areas of the gluteus maximus. Note that 3 electrodes were inserted in each muscle in cases 4 and 5; the cross-sectional areas increased significantly in these cases (paired $t$ test, $P<0.05)(1 \mathrm{~L}=$ casel, left. $1 \mathrm{R}=$ casel, right $)$

CT numbers in the gluteus medius $(P<0.01)$, the iliopsoas $(P<0.05)$, and the paraspinal muscles $(P<0.01)$ showed a significant increase using the paired $t$ test, while other muscles did not show any significant difference (Figure 5). It was generally found, however, that CT numbers did not recover to within the normal range in cases 1,3 , and 5 , where the time since injury was over 1 year; the normal range for the lower extremities was $38-74$ according to the data of Bulcke et al. ${ }^{11}$

Muscle torque measured by Cybex II and muscle force measured with the help of a strain-gauge transducer generally increased during the time course (Figure 6). Table 3 shows the muscle torque, the 


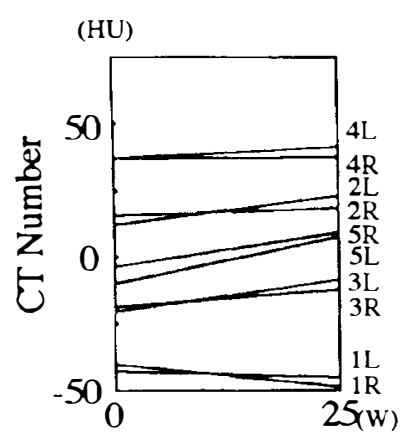

Gluteus maximus

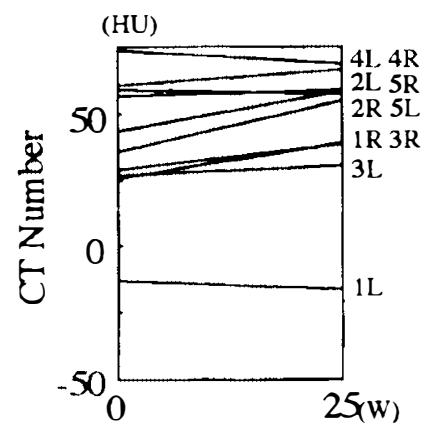

Extensors in lower leg

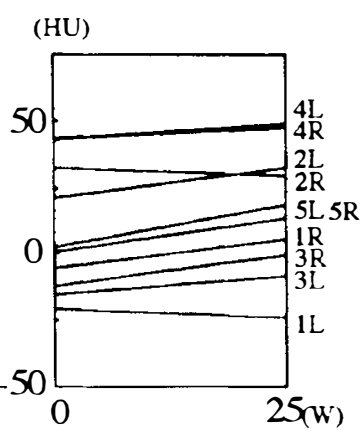

Gluteus medius

(HU)

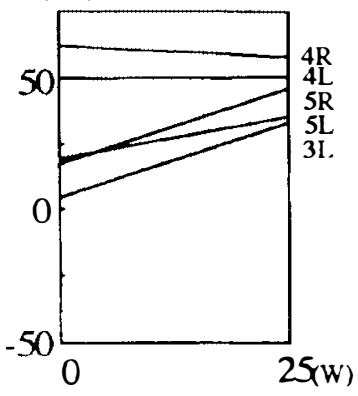

Iliopsoas

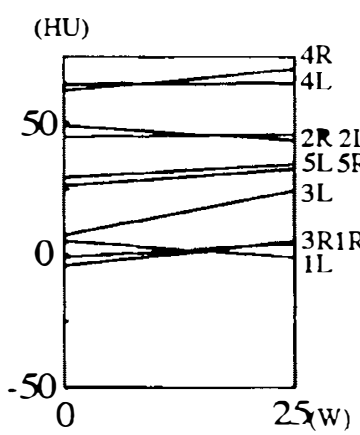

Quadriceps femoris (HU)

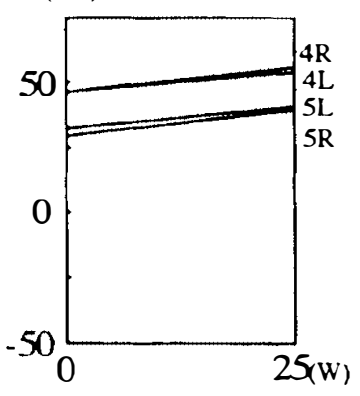

Paraspinal muscles

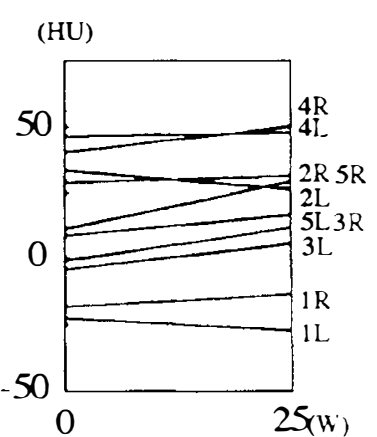

Long head of biceps femoris

Figure 5 Changes in the CT number up to 25 weeks after TES. ( $1 \mathrm{~L}=$ case 1 , left. $1 \mathrm{R}=$ case 1 , right)

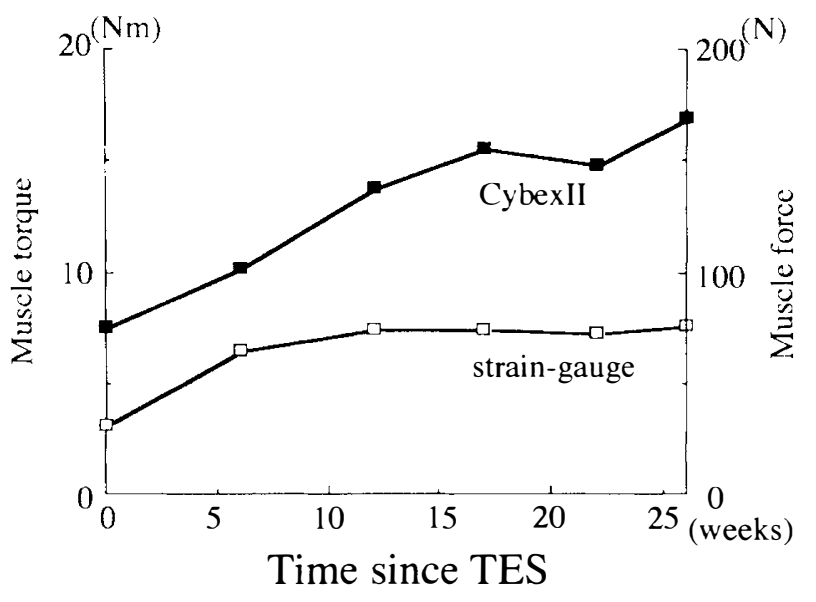

Figure 6 Changes in muscle torque and muscle force in left quadriceps femoris in case 3 measured by Cybex II and strain-gauge transducer, respectively

muscle force, and MMT at both the beginning of TES and 6 months later. Muscle torque increased from 1.7 times to 5.8 times and the maximal torque was $43.92 \mathrm{Nm}$ in the right quadriceps femoris of case 4 . Muscle force increased from 2.1 times to 5.1 times and the maximal force was $180.40 \mathrm{~N}$ in the right quadriceps femoris of case 4. Although the muscle force in the right quadriceps femoris in case 3 was too small to measure using Cybex II, it was possible to measure it with the strain-gauge transducer. Regarding MMT, the muscle force which was greater than poorminus before TES increased significantly after TES using the Wilcoxon signed-ranks test $(P<0.001)$; it increased to better than poor when the initial muscle force was greater than poor-minus, while it increased to better than good when the initial force was greater than fair. However, when the initial muscle force was only a trace, it did not show a significant change using the Binominal distribution $(P<0.05)$.

\section{Discussion}

CT, Cybex II, the strain-gauge transducer, and MMT are commonly used to evaluate muscle force. The CT scan of the muscles shows a muscle-mass increase, and suggests an increase of muscle force; however, this is an indirect monitoring method. Cybex II and the straingauge transducer measure the muscle force directly, but cannot measure all of the muscles. In addition, Cybex II cannot measure a small muscle force, while the strain-gauge transducer is a complicated method. MMT is easy to perform, but is subjective to a certain extent. ${ }^{10}$ We used all four tests to evaluate total muscle force.

It is widely accepted that muscle force is proportional to the muscle cross-sectional area and that there is at least a significant positive correlation between muscle force and cross-sectional areas. ${ }^{12}$ In complete paralysis, however, muscle force is always zero without 
Table 3 Muscle torque measured by Cybex II, muscle force measured by strain-gauge transducer and MMT before and after TES

\begin{tabular}{|c|c|c|c|c|c|c|c|c|c|}
\hline \multirow[b]{2}{*}{ Case } & \multirow[b]{2}{*}{ Muscle } & \multicolumn{3}{|c|}{ Muscle torque $(\mathrm{Nm})$} & \multicolumn{3}{|c|}{ Muscle force $(N)$} & \multicolumn{2}{|c|}{$M M T$} \\
\hline & & Initial & Follow-up & Ratio & Initial & Follow-up & Ratio & Initial & Follow-up \\
\hline \multirow[t]{2}{*}{1} & Rt. Biceps femoris & 2.68 & 5.44 & 2.0 & & & & $\mathrm{P}-$ & $\mathbf{P}+$ \\
\hline & Lt. Biceps femoris & 0 & 0 & - & & & & $\mathrm{T}$ & $\mathrm{T}$ \\
\hline \multirow[t]{4}{*}{2} & Rt. Biceps femoris & 3.21 & 10.14 & 3.2 & & & & $\mathrm{P}-$ & $\mathrm{P}+$ \\
\hline & Lt. Biceps femoris & 1.41 & 4.38 & 3.1 & & & & $\mathrm{P}_{-}$ & $\mathrm{P}$ \\
\hline & Rt. Tibialis anterior & 3.57 & 11.77 & 3.3 & & & & G & $\mathrm{N}-$ \\
\hline & Lt. Tibialis anterior & 1.22 & 7.06 & 5.8 & & & & G- & $\mathrm{G}+$ \\
\hline \multirow[t]{6}{*}{3} & Rt. Quadriceps femoris & 0 & 0 & - & 0.23 & 0.67 & 2.9 & $\mathrm{~T}$ & $\mathrm{~T}$ \\
\hline & Lt. Quadriceps femoris & 7.57 & 16.96 & 2.2 & 31.58 & 76.30 & 2.4 & $\mathrm{~F}$ & $\mathrm{G}+$ \\
\hline & Rt. Biceps femoris & & & & 0 & 0 & - & $\mathrm{T}$ & $\mathrm{T}$ \\
\hline & Lt. Biceps femoris & & & & 0.40 & 2.05 & 5.1 & $\mathrm{~T}$ & $\mathrm{~T}$ \\
\hline & Rt. Tibialis anterior & 1.03 & 4.98 & 4.8 & & & & G- & G \\
\hline & Lt. Tibialis anterior & 5.19 & 11.01 & 2.1 & & & & G & $\mathrm{N}-$ \\
\hline \multirow[t]{4}{*}{4} & Rt. Quadriceps femoris & 25.38 & 43.92 & 1.7 & 83.30 & 180.40 & 2.2 & G & $\mathrm{G}+$ \\
\hline & Lt. Quadriceps femoris & 5.47 & 15.12 & 2.8 & 32.66 & 86.21 & 2.6 & $\mathrm{~F}$ & G \\
\hline & Rt. Biceps femoris & & & & 2.85 & 6.11 & 2.1 & $\mathrm{P}-$ & $\mathrm{P}+$ \\
\hline & Lt. Biceps femoris & & & & 0 & 0 & - & $\mathrm{T}$ & $\mathrm{T}$ \\
\hline \multirow[t]{4}{*}{5} & Rt. Quadriceps femoris & 19.01 & 40.90 & 2.2 & 22.23 & 88.59 & 4.0 & G & $\mathrm{G}+$ \\
\hline & Lt. Quadriceps femoris & 9.06 & 25.97 & 2.9 & 19.31 & 57.75 & 3.0 & $\mathrm{~F}$ & $\mathrm{G}$ \\
\hline & Rt. Tibialis anterior & 2.76 & 4.46 & 1.6 & & & & G- & $\mathrm{G}$ \\
\hline & Lt. Tibialis anterior & 1.22 & 5.54 & 4.5 & & & & G- & G \\
\hline
\end{tabular}

electrical stimulation. The cross-sectional areas of all muscles showed a significant increase after TES. We also recognized an increase in muscle force measured by other methods. These results suggest that muscle force increases following TES when there has been an increase in the cross-sectional area of muscle.

The CT number is used to obtain a qualitative assessment of muscle composition; ${ }^{12}$ low-density tissue is made up of fat cells. ${ }^{13}$ We believe TES should be started as early as possible following the onset of paralysis in order to maintain muscle quality. This is because $\mathrm{CT}$ numbers are found not to recover within a normal range after TES when those numbers are below the normal range at the beginning of TES.

The measurements by Cybex II and with the help of the strain-gauge transducer demonstrated that muscle force increases several times after TES, but that the trace muscle on MMT remained a trace. We believe that the trace muscle is not useful for practical applications.

We can roughly predict the muscle force following TES after observing the muscle force at the beginning of TES. If we intend to restore muscle function in paraplegic patients using FES, a muscle force greater than a poor-minus level on MMT is necessary to accept an electrode when electrodes are being inserted in the operating room, because a minimum poor level is necessary to reconstruct motion in the lower extremities. On the other hand, anti-gravity muscles like the quadriceps femoris, that must have a greater than fair level of muscle force when electrically stimulated in the operating room, to increase to a greater than good level of muscle force. We believe that a greater than good level of muscle force is necessary for anti-gravity muscles.
The reasons for the differences in increased ratios in the cross-sectional area of muscle, the CT number, muscle torque, and muscle force in each muscle are: (1) The possibility of peripheral nerve injury. Crago et al. ${ }^{14}$ have demonstrated that stimulation takes place primarily by excitation of the intramuscular nerves, and that only $3-7 \%$ of the muscle twitch force is produced by direct stimulation of muscle fibers. If peripheral nerve injury is severe, muscle force is considered very small, since it is only produced by direct stimulation of muscle fibers. It is possible that there is peripheral nerve damage in cases 1,2 , and 3 , because the injury levels are in the thoraco-lumbar region; (2) The different patterns of nerve distribution in the muscles. The quadriceps femoris muscle is innervated directly by both the femoral nerve and intramuscular branches of the femoral nerves, while the gluteus maximus, iliopsoas etc. are stimulated only by intramuscular branches of nerves. The gluteus medius and extensors in the lower leg are stimulated directly by a main nerves. Since the inferior gluteal nerve to the gluteus maximus muscle rapidly divides into a number of branches, one electrode is not enough to contract all of the muscle fibers; several electrodes are necessary to induce sufficient muscle force in the gluteus maximus or the electrode must be placed prior to the nerve division; (3) Implantation techniques. Sometimes we cannot insert the electrodes into the best place; (4) The possibility of breakage, movement, or changes in electrode impedance. Although the rate of breakdown of our electrodes has been very low ${ }^{8}$ such problems may occur.

It is widely accepted that electrical stimulation does increase voluntary muscle force. ${ }^{3,5,6}$ Our study 
suggests that electrical stimulation also increases the muscle force of complete paralyzed muscles during electrical stimulation.

\section{Acknowledgements}

The authors wish to thank the doctors at the Department of Radiology, Akita University School of Medicine for helping with CT scanning.

\section{References}

1 Shimada Y, Sato K, Abe E, Kagaya H, Ebata K, Oba M, Arai M, Sato M, Obinata G. Restoration of locomotion in paraplegics by functional electrical stimulation. Proc 1st Intern FES Symp 1992; $110-114$.

2 Peckham PH, Mortimer JT, Marsolais EB. Alteration in the force and fatigability of skeletal muscle in quadriplegic humans following exercise induced by chronic electrical stimulation. Clin Orthop 1976; 114: 326-334.

3 Merletti R, Zelaschi F, Latella D, Galli M, Angeli S, Sessa MB. A control study of muscle force recovery in hemiparetic patients during treatment with functional electrical stimulation. Scand $J$ Rehab Med 1978; 10: 147-154.

4 Marsolais EB and Kobetic R. Functional walking in paralyzed patients by means of electrical stimulation. Clin Orthop 1983; 175: $30-36$.
5 Stefanovska A and Vodovnik L. Change in muscle force following electrical stimulation. Scand J Rehab Med 1985; 17: 141-146.

6 Delitto A and Snyder-Mackler L. Two theories of muscle strength augmentation using percutaneous electrical stimulation. Phys Ther 1990; 70: $158-164$.

7 Marsolais EB and Kobetic R. Functional electrical stimulation for walking in paraplegia. J Bone Joint Surg 1987; 69-A: 728-733.

8 Handa Y, Hoshimiya N, Iguchi Y, Oda T. Development of percutaneous intramuscular electrode for multichannel FES system. IEEE Trans Biomed Eng 1989; 36: 705-710.

9 Hoshimiya N, Naito A, Yajima M, Handa Y. A multichannel FES system for the restoration of motor functions in high spinal cord injury patients: A respiration-controlled system for multijoint upper extremity. IEEE Trans Biomed Eng 1989; 36: 754-760.

10 Kendall FP and McCreary EK. Muscles, testing and function. 3rd ed. Williams \& Wilkins, Baltimore. 1983.

11 Bulcke JA, Termote J-L, Palmers Y, Crolla D. Computed tomography of the human skeletal muscular system. Neuroradiology 1979; 17: 127-136.

12 Maughan RJ, Watson JS, Weir J. Strength and cross-sectional area of human skeletal muscle. J Physiol 1983; 338: 37-49.

13 Termote J-L, Baert A, Crolla D, Palmers Y, Bulcke JA. Computed tomography of the normal and pathologic muscular system. Radiology 1980; 137: 439-444.

14 Crago PE, Peckham PH, Mortimer JT, Meulen JP. The choice of pulse duration for chronic electrical stimulation via surface, nerve, and intramuscular electrodes. Ann Biomed Eng 1974; 2: $252-264$. 